\title{
Analysis of the linear relationship between asymmetry and magnetic moment at the $M$ edge of $3 d$ transition metals
}

\author{
Somnath Jana $\odot,{ }^{1}$ R. S. Malik $\odot,{ }^{1}$ Yaroslav O. Kvashnin, ${ }^{1}$ Inka L. M. Locht, ${ }^{1}$ R. Knut, ${ }^{1}$ R. Stefanuik, ${ }^{1}$ Igor Di Marco $\odot,{ }^{1,2,3}$ \\ A. N. Yaresko, ${ }^{4}$ Martina Ahlberg, ${ }^{5}$ Johan Åkerman, ${ }^{5}$ Raghuveer Chimata, ${ }^{1,6}$ Marco Battiato ${ }^{\circledR},{ }^{7}$ Johan Söderström, ${ }^{1}$ \\ Olle Eriksson, ${ }^{1,8}$ and Olof Karis ${ }^{1}{ }^{1}$ \\ ${ }^{1}$ Department of Physics and Astronomy, Uppsala University, Box 516, SE-75120 Uppsala, Sweden \\ ${ }^{2}$ Asia Pacific Center for Theoretical Physics, Pohang, Gyeonbuk 790-784, Republic of Korea \\ ${ }^{3}$ Department of Physics, POSTECH, Pohang, Gyeonbuk 790-784, Republic of Korea \\ ${ }^{4}$ Max-Planck-Institut für Festkörrperforschung, Heisenbergstrasse 1, D-70569 Stuttgart, Germany \\ ${ }^{5}$ Department of Physics, University of Gothenburg, SE-41296 Gothenburg, Sweden \\ ${ }^{6}$ Argonne National Laboratory, Lemont, Illinois 60439, USA \\ ${ }^{7}$ Nanyang Technological University, 21 Nanyang Link, 637371 Singapore, Singapore \\ ${ }^{8}$ School of Science and Technology, Örebro University, SE-70182 Örebro, Sweden
}

(Received 13 August 2019; revised manuscript received 28 November 2019; accepted 23 December 2019; published 20 February 2020)

\begin{abstract}
The magneto-optical response of $\mathrm{Fe}$ and $\mathrm{Ni}$ during ultrafast demagnetization is studied experimentally and theoretically. We have performed pump-probe experiments in the transverse magneto-optical Kerr effect (T-MOKE) geometry using photon energies that cover the $M$ absorption edges of Fe and Ni between 40 and $72 \mathrm{eV}$. The magnetic asymmetry was obtained by forming the difference of reflected intensities obtained for two opposite orientations of the sample magnetization. Density functional theory (DFT) was used to calculate the magneto-optical response of different magnetic configurations, representing different types of excitations: long wavelength magnons, short wavelength magnons, and Stoner excitations. In the case of Fe, we find that the calculated asymmetry is strongly dependent on the specific type of magnetic excitation. Our modeling also reveals that during remagnetization $\mathrm{Fe}$ is, to a reasonable approximation, described by magnons, even though small nonlinear contributions could indicate some degree of Stoner excitations as well. In contrast, we find that the calculated asymmetry in $\mathrm{Ni}$ is rather insensitive to the type of magnetic excitations. However, there is a weak nonlinearity in the relation between asymmetry and the off-diagonal component of the dielectric tensor, which does not originate from the modifications of the electronic structure. Our experimental and theoretical results thus emphasize the need to consider a coupling between asymmetry and magnetization that may be more complex than a simple linear relationship. This insight is crucial for the microscopic interpretation of ultrafast magnetization experiments.
\end{abstract}

DOI: 10.1103/PhysRevResearch.2.013180

\section{INTRODUCTION}

Since the first experimental observation of ultrafast demagnetization in Ni [1], ultrafast magnetodynamics has attracted significant interest, due to the perspective of controlling the magnetization on subpicosecond timescales [2-15]. These studies have led to the discovery of novel effects such as alloptical switching $[16,17]$ and ultrafast spin currents [18-22]. Probing the magnetic state in condensed matter using light has been a widespread approach for many decades. This can be achieved in either reflectivity measurements, based on the magneto-optical Kerr effect (MOKE), or in transmission

Published by the American Physical Society under the terms of the Creative Commons Attribution 4.0 International license. Further distribution of this work must maintain attribution to the author(s) and the published article's title, journal citation, and DOI. measurements using the Faraday effect or magnetic circular dichroism. The relation between the magneto-optical response of the material in the subpicosecond timescale and its instantaneous magnetization has been investigated in the past for various geometries [23-31], but, as illustrated below, no clear conclusion was reached and the topic still remains controversial. Using light as both pump and probe provides the possibility of studying ultrafast magnetic processes in the femtosecond time regime. Typically, an intense ultrashort (tens of femtoseconds) laser pulse is used to excite the magnetic sample and then the response is measured by another temporally short but less intense optical pulse, so that the latter has an insignificant effect on the magnetic state. Most ultrafast magnetization studies have been performed using light in the visible regime because it is easily accessible. However, during the past few decades, high photon energies have become available through high-order harmonic generation (HHG) sources reaching extreme ultraviolet (EUV) and by synchrotrons facilities providing energies in the soft $\mathrm{x}$-ray regime. The main 
advantage of using EUV or higher photon energies is that one can reach absorption edges that provide higher magnetic contrast and elemental selectivity. With the access to HHG sources, element-specific magnetization dynamics becomes possible in laboratory-based setups, where the magnetic state is probed in the transverse MOKE (T-MOKE) geometry using linearly polarized XUV. In the T-MOKE geometry, the magnetization is perpendicular to the plane of incidence and also to the polarization of the light. This geometry results in a change of intensity of the reflected light when the magnetization changes, unlike the longitudinal and the polar Kerr effect, where the polarization of the reflected light changes [32]. The T-MOKE geometry is highly advantageous for high-energy photons as circularly polarized light is not easily accessible for high-order harmonics and polarimetry is not easily performed due to the limited availability of optical components at high photon energies. The T-MOKE signal is commonly assumed to be approximately proportional to the magnetization of the probed sample (see, e.g., Refs. [33,34]). Since the signal is strongly enhanced near the absorption edge of a given magnetic element, the proportionality is expected to be dominated by the local magnetization of that element at the resonant energies [8,30,33,34].

Similar to previous studies [8,30,33,34], we have measured the reflected intensity of $p$-polarized light at angles close to the Brewster angle. The measurements were made for two opposite magnetization directions (yielding $I_{+}$and $I_{-}$) and the asymmetry, $A(E)$, in the measurement was defined as $[30,32,34]$

$$
A(E)=\frac{I_{+}(E)-I_{-}(E)}{I_{+}(E)+I_{-}(E)},
$$

where $E$ represents the energy (frequency) of the probe light. Assuming that the off-diagonal components to the dielectric tensor are small compared to the Fresnel coefficient, it was shown in Ref. [34] that

$$
A(E) \approx 2 \operatorname{Re}\left[\frac{\sin (2 \theta) \epsilon_{x y}(E)}{n^{4}(E) \cos ^{2} \theta-n^{2}(E)+\sin ^{2} \theta}\right]
$$

where $n(E)$ is the complex refractive index, $\epsilon_{x y}$ is the offdiagonal component of the dielectric tensor $\boldsymbol{\epsilon}$, and $\theta$ is the angle of incidence relative to the surface normal. In this equation, the refractive index $n$ depends on the energy of the light. Owing to the Onsager relationship, $\epsilon_{x y}$ changes sign when the magnetic moment of the sample is reversed. It thus follows from Eq. (2) that the asymmetry, $A(E)$, is an odd function in the magnetization $\mathbf{M}$ for any energy $E$. Often a linear relationship is assumed, but one should note that this is not always a valid approximation, as will be demonstrated here. The reason can be twofold: There are either higher order contributions in $\mathbf{M}$ influencing $\epsilon_{x y}$ (via changes in the electronic structure in the excited states) or additional contributions to $A(E)$ which are not accounted for by the approximated Eq. (2). The way this is analyzed here is by making an assumption that $\epsilon_{x y} \propto$ M. From Eq. (2), it then follows that

$$
A(E) \approx K(E) \mathbf{M},
$$

where $K(E)$ is an energy-dependent proportionality constant. If the relationship of Eq. (3) holds, one should be able to follow the asymmetry of the reflected light for two different photon energies, $E$ and $E^{\prime}$, and the ratios in asymmetry would be $\frac{K(E)}{K\left(E^{\prime}\right)}$, i.e., a constant that does not depend on M. Any deviation from this behavior in, e.g., a pump-probe experiment, must indicate that higher order contributions in $\mathbf{M}$ influence $\epsilon_{x y}$, demonstrating complexities in the band structure. In this study, we will undertake such an analysis and demonstrate that one can use this relationship between $A(E)$ and $\mathbf{M}$ to draw conclusions about the microscopic mechanisms of the magnetization dynamics of such experiments. It is important to stress that nonmagnetic contributions due to the transient variation of the refractive index by the nonequilibrium hotelectron distribution are negligible for the chosen experimental geometry [30].

Ultrafast demagnetization has previously been studied in a Co film, by means of measuring the asymmetry from the T-MOKE signal at $M$ edge $[35,36]$. The results conclude that both ultrafast magnon generation and transient reduction of the exchange splitting are responsible for the ultrafast demagnetization. Analysis at two different pump-probe delays suggests that the ultrafast magnon generation is dominant during subpicosecond timescales, while the transient reduction of the exchange splitting persists for several picoseconds. This is rather counterintuitive from the total energy consideration. This scenario was supported by an independent photoemission study [37]. The pump-probe response of Co was also investigated by atomistic spin-dynamics simulations [38], in which the relevant information is obtained from $a b$ initio electronic structure theory (see also Ref. [39]), which argued that the magnetic response should be dominated by transverse spin disorder, following the two-temperature model [6].

In a separate work on Fe, by means of magneto-optical and reflectivity spectroscopy, transversal spin fluctuation was found to be the dominant mechanism rather than a change in exchange splitting [29], which is consistent with results from atomistic spin-dynamics simulations, although the lower range for appropriate timescales for such simulations is of order of a few hundreds fs [40]. Recently, the problem of laser-induced demagnetization has been addressed by timedependent density functional theory (TD-DFT) [41]. This is a rigorous approach, which in principle describes the evolution of the electron density in the first moments after the optical irradiation. Practical implementations are naturally associated with approximations, and the results of TD-DFT calculations have to be critically examined and compared to experiments. The results obtained for three elemental ferromagnets (Fe, $\mathrm{Co}, \mathrm{Ni}$ ) reveal similar trends of dynamics, where the demagnetization is primarily caused by the spin-flip electron excitation $[42,43]$. Short wavelength magnons are predicted to be irrelevant at the ultrashort timescales, but the predictive power of these studies is limited by the small size of computationally treatable supercells. In addition, it is reported that the demagnetization is enhanced at the surface of a material as compared to the bulk [44].

The examples above serve to illustrate that a firm theoretical understanding of pump-probe experiments in the fs timescale is still evolving and that additional analysis and measurements are needed. To this end, it is important to clarify the proportionality relations described in Eq. (1), something we attempt to do here. In this work, we have studied the 

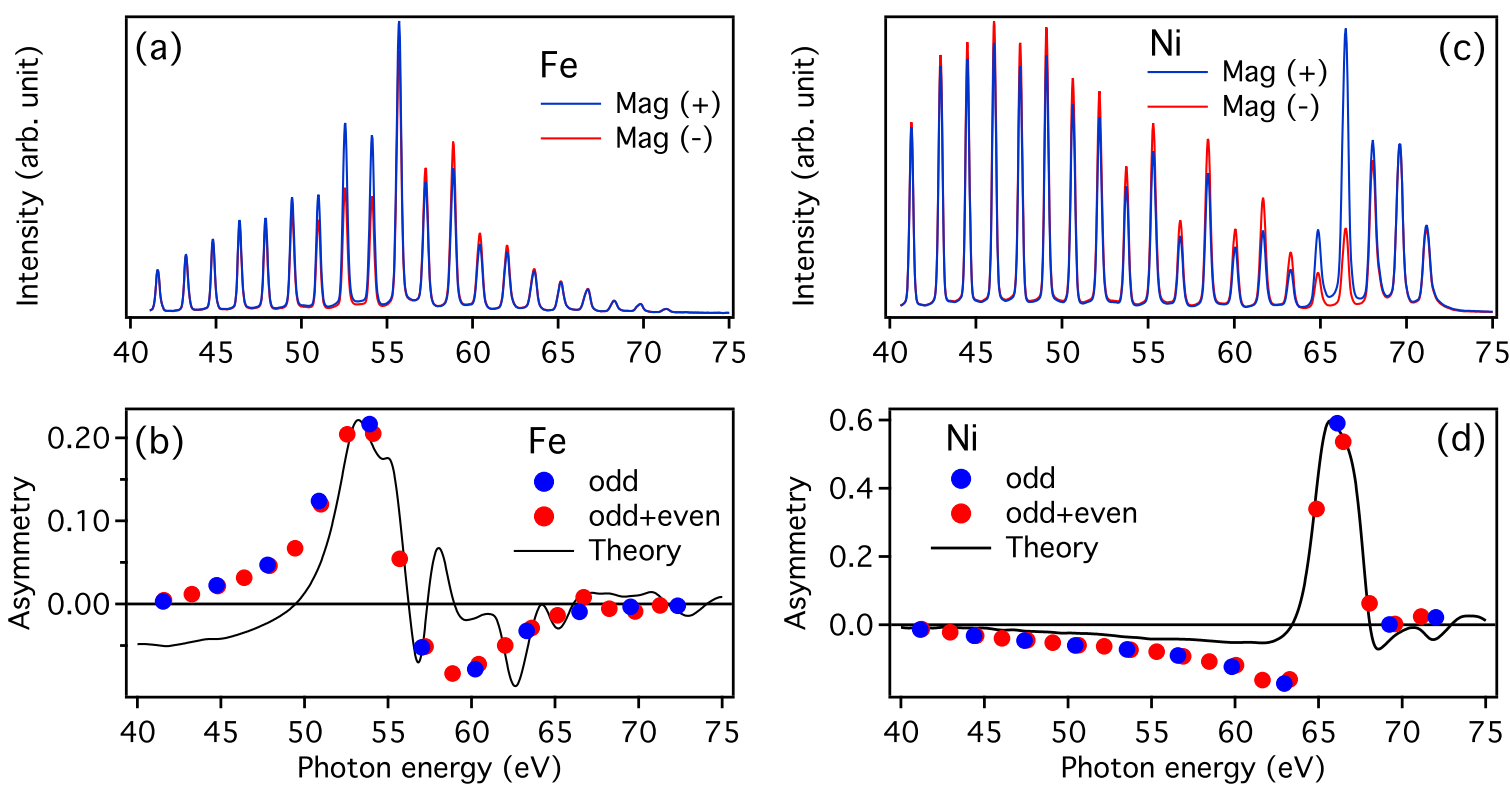

FIG. 1. (a) The reflected harmonic intensities $I_{ \pm}(E)$ for Fe. (b) The corresponding magnetic asymmetry for Fe, using "odd" and "odd and even" harmonics as explained in the text. (c) The reflected harmonic intensities $I_{ \pm}(E)$ for Ni. (d) The corresponding magnetic asymmetry for Ni, using "odd" and "odd and even" harmonics as explained in the text. Ground-state theoretical asymmetry spectra are plotted in panels (b) and (d). See the text for computational details.

magnetic asymmetry of ferromagnetic films $(\mathrm{Fe}, \mathrm{Ni})$ in the T-MOKE geometry with probing photon energies ranging between 40 and $72 \mathrm{eV}$. The dynamics of the observed magnetic signal at different photon energies is compared to $a b$ initio derived theoretical spectra, corresponding to several possible types of magnetic excitations. It is found that $\mathrm{Fe}$ exhibits strong nonlinear effects in the energy-dependent asymmetry during the demagnetization. However, during the remagnetization mainly a linear response is found, which indicates that the system contains mainly longer wavelength magnons. $\mathrm{Ni}$ exhibits a more or less proportional relation between asymmetry and magnetization for all types of excitations considered in our modeling. This is also reflected in the experimental data that only show a weak energy dependence of the asymmetry. We note that there is an additional nonlinearity between $A$ and $\epsilon_{x y}$, which is more pronounced in $\mathrm{Ni}$ than in $\mathrm{Fe}$.

\section{EXPERIMENT}

\section{A. Experimental setup}

The experiments are performed at the HELIOS Laboratory, Uppsala University, Sweden. The near-infrared (NIR) pump pulse has a wavelength of $\approx 800 \mathrm{~nm}(\approx 1.5 \mathrm{eV})$ and a pulse length of $\approx 35 \mathrm{fs}$. The broadband EUV probe pulse has energies between 40 and $72 \mathrm{eV}$ and a pulse length of $\approx 20 \mathrm{fs}$ [45-47]. Since the NIR pump and the EUV pulse are generated from the same laser pulse, the temporal jitter between the NIR and EUV is eliminated and hence the temporal resolution is determined by the pulse widths. The measurement geometry is described in detail in Ref. [47]. Both the p-polarized EUV and the NIR are focused on the sample at $45^{\circ}$ incidence angle, i.e., near the Brewster angle for EUV photons, that gives optimal magneto-optical contrast due to minimized nonmagnetic reflectivity at this angle. The reflected EUV probe is dispersed by a subsequent spectrometer, while the reflected
NIR pump is absorbed by a $200-\mu$ m-thick $\mathrm{Al}$ foil [47]. In the T-MOKE geometry, the sample is magnetized perpendicularly to the plane of incidence of the incoming $p$-polarized light. The reflectivity for opposite magnetization directions $( \pm \mathbf{M})$ is measured as a function of the harmonic energy $E$ and is labeled $I_{ \pm}(E)$. A measured energy-dependent background (dark noise in the detector system) has been subtracted from all spectra. For time-resolved pump-probe measurements, the spectra are composed of odd harmonics of the fundamental light, thus having a splitting of $\approx 3.1 \mathrm{eV}$. For the static case, HHG spectra containing all harmonics (even and odd) were obtained by mixing the fundamental light with its second harmonic in the HHG process [48], as shown in Figs. 1(a) and $1(\mathrm{c})$.

From a practical point of view, theory of the asymmetry function has to use a slightly modified version of Eq. (1), i.e.,

$$
A(E)=\frac{I_{+}(E)-I_{-}(E)}{I_{+}(E)+I_{-}(E)+\Gamma},
$$

where Eq. (1) has been augmented by a unit less constant $\Gamma$ in the denominator, to account for effects that are not included in the theoretical calculations, e.g., the background signal. We will discuss this point in more detail in Sec. III.

Two samples with elemental films of $\mathrm{Fe}$ and $\mathrm{Ni}$, respectively, were used in this investigation. A 100-nm Ni film was deposited on a $\mathrm{Si}$ wafer by means of magnetron sputtering. Upon exposure to air, this sample forms a native oxide layer of about $1 \mathrm{~nm}$ thickness [49,50], which acts as a protection against further oxidation. Similarly, a $100-n m$ Fe film was sputtered on a Si wafer and subsequently capped with a 5-nm $\mathrm{Cu}$ layer to prevent oxidation of the Fe film. Unlike $\mathrm{Ni}$, the Fe layer becomes fully oxidized when exposed to air unless it is protected by a capping layer. Both samples possess an in-plane anisotropy with a coercivity of $\leqslant 50$ Oe. The external magnetic field was set to $\approx \pm 800 \mathrm{Oe}$, and thus the films were 

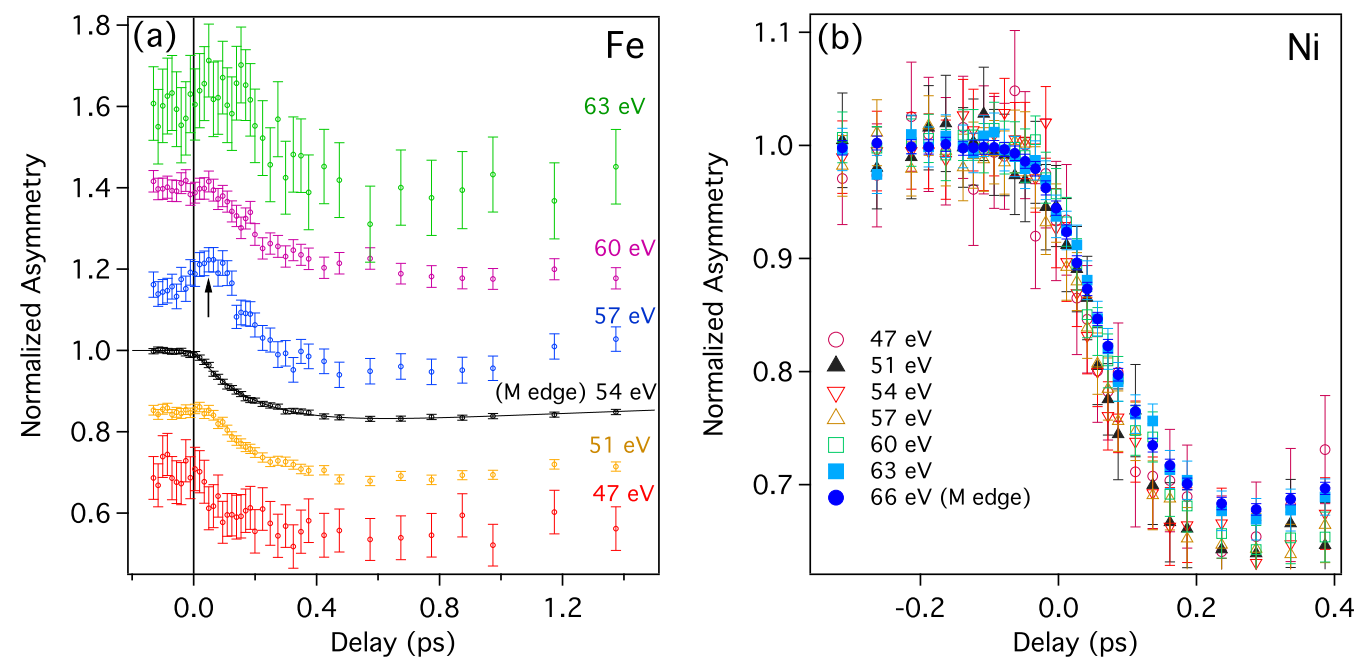

FIG. 2. (a) Measured asymmetry for the bcc Fe sample at different harmonic energies, as a function of delay time between pump and probe. There are strong irregularities in the energy dependent asymmetry data for Fe, highlighted by the arrow ( $57 \mathrm{eV}$ ). All curves except at $54 \mathrm{eV}$ have been shifted vertically for clarity. For the $54 \mathrm{eV}$ harmonic, we also show a representative fit to the data (solid black line). (b) Measured asymmetry for the fcc Ni sample at different harmonic energies, as a function of delay time between pump and probe. No irregularities, similar to those found for $\mathrm{Fe}$, are found for $\mathrm{Ni}$.

saturated during the measurements. All the measurements were performed at room temperature. The repetition rate of the laser, and hence the XUV light, was $10 \mathrm{kHz}$. The pump fluences (for demagnetizing the samples) were set to $\approx 1.3$ and $\approx 4 \mathrm{~mJ} / \mathrm{cm}^{2}$ in cases of $\mathrm{Ni}$ and $\mathrm{Fe}$, respectively.

\section{B. Experimental results}

The difference in arrival time between the pump and probe light is controlled by a delay stage. If the probe pulse arrives before the pump pulse, the sample will be fully magnetized, resulting in a large asymmetry. The measured asymmetry $A(E)$, for fully magnetized $\mathrm{Fe}$ and $\mathrm{Ni}$ is reported in Figs. 1(b) and 1(d), respectively. As can be observed in Fig. 1(b), the asymmetry of Fe is distributed over the whole available spectrum of harmonics, but most strongly peaked at one harmonic energy. The same effect can be observed for the Ni sample, in Fig. 1(d), but the main peak is now shifted with about $\approx 12 \mathrm{eV}$ to higher photon energy. Since the energy splitting between odd harmonics is relatively large $(\approx 3.1 \mathrm{eV})$, we also present the asymmetry obtained with spectra containing both even and odd harmonics, giving a splitting of $\approx 1.55 \mathrm{eV}$. This provides a more detailed experimental determination of the asymmetry and shows that the odd harmonics, which are used for our time-resolved studies, capture the peak asymmetry of both $\mathrm{Fe}$ and $\mathrm{Ni}$ at $\approx 54$ and $\approx 66 \mathrm{eV}$, respectively. The asymmetries correspond to the average value in a range of $\pm 0.15 \mathrm{eV}$ from the harmonic peaks. If the probe arrives after the pump, the sample demonstrates a decreased asymmetry due to the ultrafast demagnetization process. This is illustrated in Figs. 2(a) and 2(b), where the asymmetries of $\mathrm{Fe}$ and $\mathrm{Ni}$ are reported as a function of the delay between pump and probe. At $t=0 \mathrm{ps}$, the pump and probe pulses are incident on the sample simultaneously. Within a time delay of less than $1 \mathrm{ps}$, the samples show a strong reduction of the asymmetry, i.e., a strong demagnetization. After the initial reduction, the asymmetries start returning to their initial value, which indicates a recovery of the sample magnetization on a longer timescale.

While it is certain that the asymmetry, up to a large degree, reflects the sample magnetization, their precise relationship is rather complex. If the asymmetry was proportional to the magnetization, we would expect the asymmetry to be independent of the photon energy of the probe pulse during the demagnetization and remagnetization process. The magnetic asymmetry of Fe during the demagnetization process is shown in Fig. 2(a) for six different photon energies. For the curve corresponding to the harmonic with the peak asymmetry $(\approx 54 \mathrm{eV})$, the reduction of the asymmetry starts earlier than for most of the curves at both higher and lower photon energies. This illustrates the complexity of identifying a relationship between magnetic asymmetry and magnetization. Analogous data for $\mathrm{Ni}$ is reported in Fig. 2(b), but, unlike for $\mathrm{Fe}$, the curves show much less variation with the photon energy.

According to the discussion around Eqs. (1)-(3), one would expect that if the measured asymmetry is proportional to the magnetization [Eq. (3)], the ratio of asymmetries detected at two different energies would be independent of the magnetic moment. In a pump-probe experiment, this would be manifested as a time-independent ratio. In order to investigate this, we have taken the ratio between all measured curves in Fig. 2, with respect to the measured asymmetry at energies close to the absorption edge $(54 \mathrm{eV}$ for bcc Fe and $66 \mathrm{eV}$ for fcc Ni). Since the asymmetry for each harmonic is independently normalized with respect to its value at $t<$ 0 (where no pump effect is present), the ratios are close to unity. We have plotted these ratios in Fig. 3, separated vertically from each other by 0.2 for better visualization. We will refer to such ratios as the magnetization-asymmetry test ratio (MAT ratio), both for the demagnetization (0-1.0 ps) and the remagnetization processes $(t \geqslant 1.0 \mathrm{ps})$. It may be noted from Fig. 3 that for fcc Ni the MAT ratio is much more constant than for bcc Fe, suggesting that for fcc Ni 

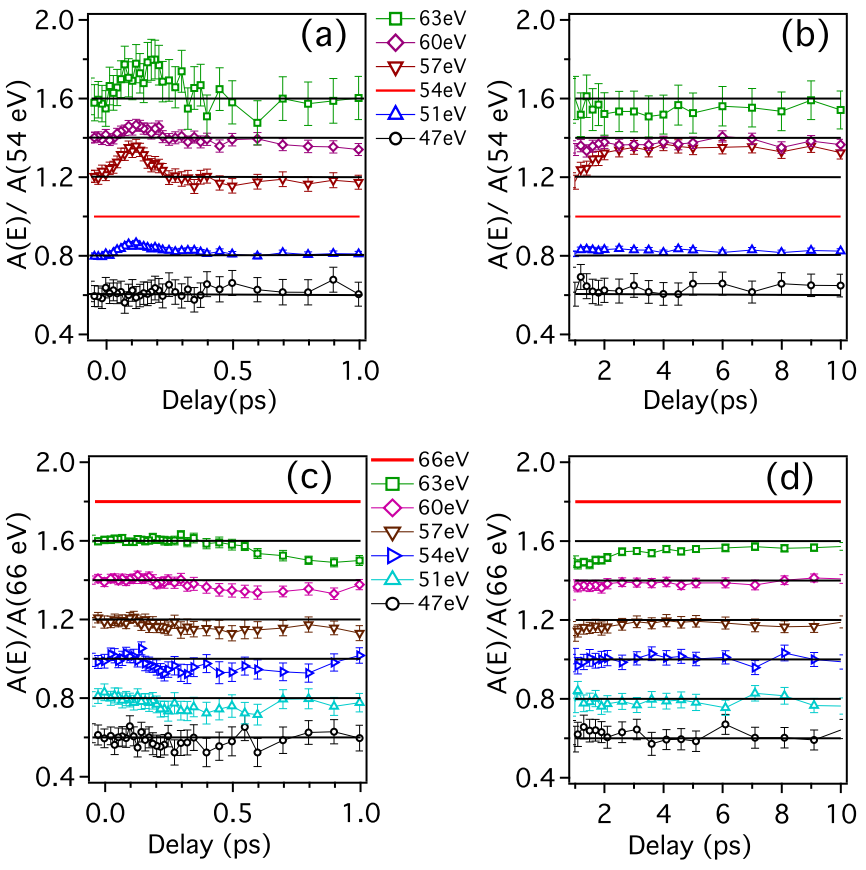

FIG. 3. The measured magnetization-asymmetry test ratio (MAT ratio; for details, see text) of bcc Fe in panels (a) and (b) and fcc Ni in panels (c) and (d) as a function of time delay. The measured data are shown in two time domains that represent the demagnetization [(a), (c)] as well as the remagnetization process [(b), (d)]. The MAT ratios are shifted from each other by 0.2 along the vertical axis (represented by the black dashed lines) for better visualization.

the measured asymmetry does indeed reflect the magnetic state to a high accuracy. However, for bcc Fe, the situation is much more complex. For this element, the demagnetization process shows a MAT ratio that clearly casts doubt on the asymmetry being proportional to the magnetic moment, since MAT ratios taken at many different energies are far from being time independent. The remagnetization process of bcc Fe at pump-probe delays longer than 2 ps is more well behaved, as suggested by fact that the corresponding MAT curves are close to constant for $t>2 \mathrm{ps}$.

\section{THEORY}

\section{A. Computational details}

To analyze the results in Figs. 2 and 3, we have performed ab initio calculations of the relationship between the magnetic asymmetry and the magnetization for $\mathrm{Fe}$ and $\mathrm{Ni}$. As recently demonstrated [31], calculations do not need to include transient effects explicitly, but can be performed in the quasistatic limit, where the sample magnetization can be treated as a constraint. In our approach, the magnetic asymmetry is calculated in two steps. First, the dielectric tensor is calculated for the bulk material from first principles by means of DFT. Thereafter, the Fresnel equations, which define the optical response of the material, are solved numerically [51,52] for a chosen sample thickness. The resulting reflectivities for the two magnetization directions are then used to obtain the magnetic asymmetry via Eq. (4), in which $\Gamma$ is a constantenergy background to take into account extrinsic contributions to the reflectivity, such as capping layer in the case of $\mathrm{Fe}$ and natural oxide surface layer in the case of $\mathrm{Ni}$. We have chosen $\Gamma$ equal to 0.0052 and 0.004 in cases of $\mathrm{Fe}$ and $\mathrm{Ni}$, respectively, in order to match the peak asymmetry between theoretical and experimental results. The same values of $\Gamma$ were then used to simulate the asymmetry for the partially demagnetized configurations.

The DFT calculations were done using the full-potential linearized augmented plane wave (FP-LAPW) method as implemented in the Elk code [53]. Both materials were considered in their ground-state structures with experimental lattice parameters equal to 5.42 a.u. and 6.66 a.u., for $\mathrm{Fe}$ and $\mathrm{Ni}$, respectively. Exchange and correlation effects were treated with the Perdew-Burke-Ernzerhof (PBE) functional [54]. ${ }^{1}$ The radii of the muffin-tin spheres $R_{M T}$ were set to 2.0 a.u. for both $\mathrm{Fe}$ and $\mathrm{Ni}$ and $3 p$ states were treated as a part of the valence band. The $k$-point grid of $30^{3} k$ points was employed together with a fairly large amount of empty states (up to $70 \mathrm{eV}$ above the Fermi level) in order to ensure the convergence of the optical properties. The dielectric tensor was calculated from the obtained band structure using standard Kubo-Greenwood formalism. The dielectric tensor was subsequently convoluted by a Gaussian of $1.2 \mathrm{eV}$ to mimic the experimental asymmetries. The corresponding calculated ground-state asymmetries are presented in Figs. 1(b) and 1(d) for $\mathrm{Fe}$ and $\mathrm{Ni}$, respectively. An energy shift of $2.0 \mathrm{eV}$ for $\mathrm{Fe}$ and $2.2 \mathrm{eV}$ for $\mathrm{Ni}$, respectively, was applied to the theoretical asymmetry spectra in order to align them to the experimental data. The origin of these shifts has been very recently identified in a mix of many-body effects, which are only roughly described in Kohn-Sham DFT, and local field effects [56]. As is clear from Figs. 1(b) and 1(d), the calculations and experiments are found to agree rather well. Theory and experiment show a smooth behavior below the peak asymmetry for both $\mathrm{Fe}$ and Ni. Also, large asymmetries are found up to $10 \mathrm{eV}$ above the peak asymmetry for Fe, even though theory indicates sharper structures in the asymmetry that are not observed experimentally. For $\mathrm{Ni}$, both theory and experiment show that the asymmetry is strongly diminished at higher energies.

As mentioned above, to investigate the relation between the magnetic asymmetry and the magnetization of the sample, we have treated the system in the quasistatic limit [31]. In this regime, the local magnetic moments are well defined and can be used to classify all possible microstates of the system. For our purposes, we have to consider only those microstates that are compatible with the decreased value of the $z$ projection of the magnetization. These states can be grouped in three families, as illustrated in Fig. 4. In Fig. 4(a), the total magnetization is decreased by shrinking the length of all atomic magnetic moments (corresponding to Stoner excitations), in Fig. 4(b) the total magnetization is decreased by exciting long wavelength spin waves, and in Fig. 4(c) the

\footnotetext{
${ }^{1}$ Additional set of calculations, performed using the functional based on local spin density approximation [55], produced nearly the same results. The two functionals result in slightly different exchange splitting of the $3 d$ band, but the overall shape of the DOS is identical. Since the dielectric tensor is only sensitive to the unoccupied part of the DOS, the final spectra of magnetic asymmetry appear slightly shifted with respect to each other but are otherwise the same.
} 


\section{(a) Stoner-like excitation}

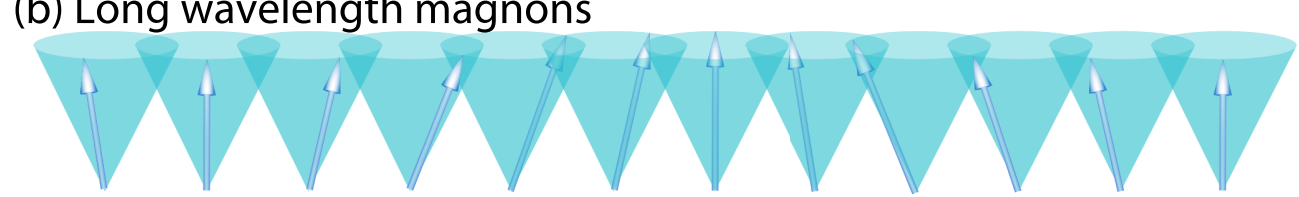

(c) Short wavelength magnons
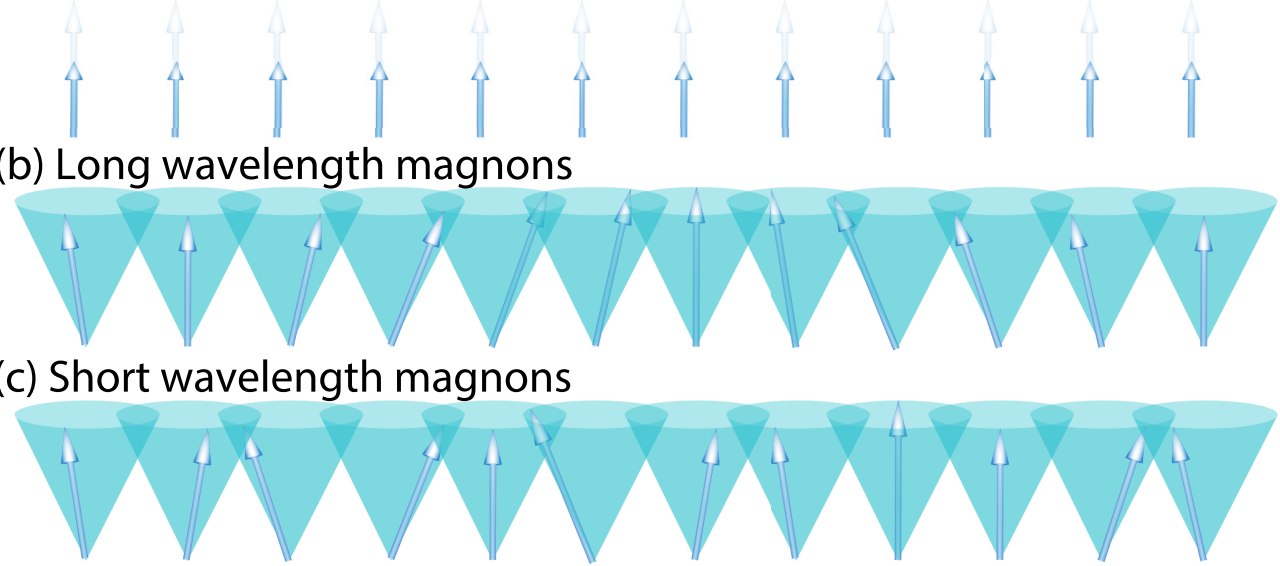

FIG. 4. Illustration of different microscopic states with decreased total magnetization. In panel (a) the length of all atomic magnetic moments are decreased (corresponding to Stoner excitations), in panel (b) the magnetization is decreased by long wavelength spin waves, and in panel (c) the atomic magnetic moments are tilted in random directions, thus representing a high density of short wavelength magnons.

atomic magnetic moments are tilted from the $\hat{z}$ axis over an angle $\Theta$ in random directions, thus representing a high density of short wavelength magnons. For these possible scenarios, we have calculated the dielectric tensor and the corresponding asymmetry for a prescribed value of $\mathbf{M}$. Each microscopic picture was modeled in the following way:

(1) Stoner-like excitations. Collinearly decreased atomic magnetic moments. The ferromagnetic atomic magnetic moments, projected onto the muffin-tin sphere, were shrunk to a certain value using constrained DFT. This approach offers a better description of the excited state than other commonly used approaches, as, e.g., a repopulation of the rigid band structure or a modification of the exchange splitting. The Kohn-Sham quasiparticles are in fact self-consistently renormalized to adjust to the constraint.

(2) Long wavelength magnon excitations. This can also be though of as a gradual tilting of atomic magnetic moments. The dielectric tensor calculated for the equilibrium ferromagnetic state was tilted by an angle $\Theta$ from the $\hat{z}$ axis, rotated by an angle $\phi$ around this axis, and then averaged over all angles $0 \leqslant \phi \leqslant 2 \pi$. This implies a simple transformation of the dielectric tensor, as explained in Appendix F of Ref. [31].

(3) High density of short wavelength magnons. This can be conceptualized as a random tilting of atomic magnetic moments. Supercells containing 16 atoms were constructed for bcc Fe and fcc $\mathrm{Ni}$, where both the direction and the magnitude of each magnetic moment were fixed. The direction of each atomic moment was constrained to an axis randomly tilted from the $\hat{z}$ axis by an angle $\Theta$, and hence the $z$ projection of all moments were the same and equal to $M_{s} \cos (\Theta)$, where $M_{s}$ is the corresponding saturation magnetization. The azimuthal angles $(\phi)$ of the axes were chosen in such a way that the total in-plane component of the magnetization cancels out. This way, the supercells are characterized by only one nonzero projection of the total moment and the symmetry of the dielectric tensor is the same as in FM state. Note that these states are supposed to provide the largest expected correction to the dielectric tensor for this type of excitations. Thus, for instance, the case of coherent short wavelength magnons was not considered, since the results are expected to be in between the present scenario and the previous one.
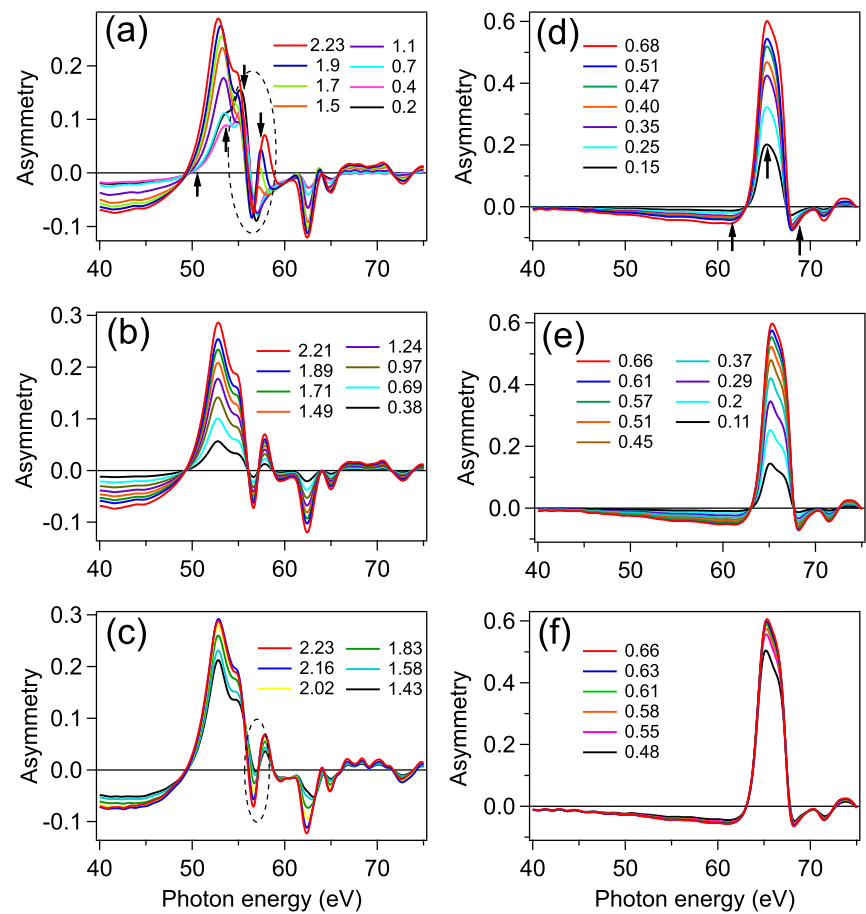

FIG. 5. Theoretical asymmetries for three different types of magnetic excitations, namely, Stoner-like excitation where moments are decreased collinearly [(a), (d)], long wavelength magnon excitations (gradually tilted magnetic moments) [(b), (e)], and a high density of short wavelength magnons (randomly tilted magnetic moments) [(c), (f)] are plotted for different magnetization in panels (a), (b), and (c) for Fe and panels (d), (e), and (f) for Ni. The value of the reduced magnetic moment for each configuration is given in the upper right part of each plot in units of $\mu_{B}$. The regions indicated by dashed ellipses are discussed in the text. The arrows indicate the energies used to calculate the real and imaginary parts of $\epsilon_{x y}$, presented in Fig. 7. 

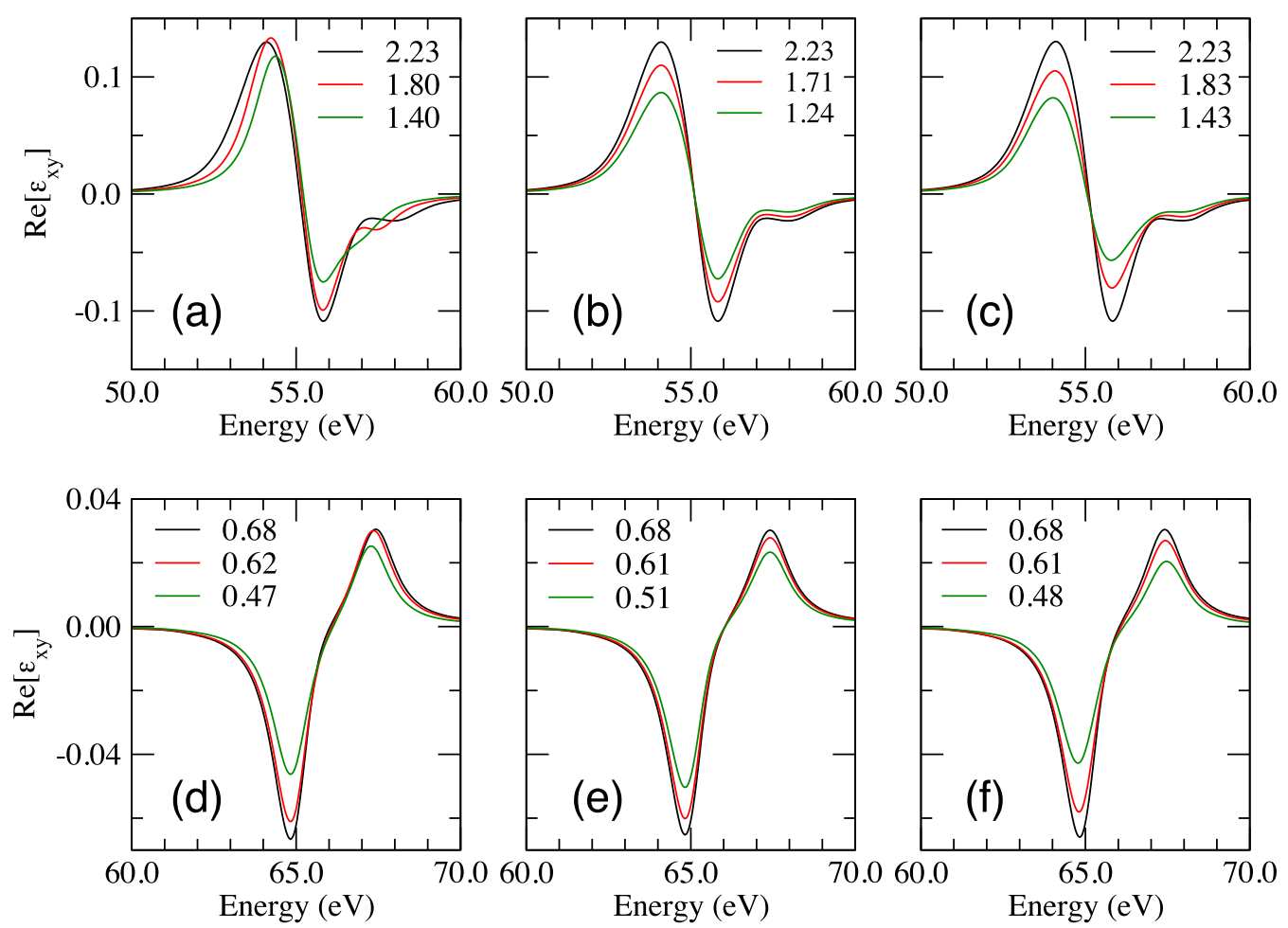

FIG. 6. Calculated real part of $x y$ component of the dielectric tensor $\left(\epsilon_{x y}\right)$ for several values of reduced magnetization (in $\left.\mu_{B}\right)$, for Stoner-like excitation in panels (a) and (d), long wavelength magnon excitations in panels (b) and (e), and a high density of short wavelength magnons in panels (c) and (f). Results for Fe are in panels (a)-(c) and for Ni in panels (d)-(f). The imaginary part of $\epsilon_{x y}$ can be obtained from the Kramers-Kronig transformation.

\section{B. Magnetic asymmetry for decreased total magnetization}

In Figs. 5(a), 5(b), and 5(c), we present the calculated asymmetries of $\mathrm{Fe}$ for Stoner-like excitations, long wavelength magnons, and short wavelength magnons, respectively. Calculations for both the long wavelength and the short wavelength magnon excitations show a monotonic decrease in asymmetry signal with decreasing magnetization for all energies.

The situation is different for the case of Stoner-type excitation, which exhibits shifts and significant changes so that a monotonic decrease is no longer observed. This is particularly clear at the energies highlighted with a dashed ellipse. Even though the modeling of short wavelength magnons exhibits a monotonic behavior, there are energy regions, primarily above the peak asymmetry, that show irregular variations with magnetization. The most conspicuous behavior is highlighted with a dashed ellipse and it is clear that for these energies, the asymmetry and magnetic moment are not linearly proportional to each other.

The overall behavior of Figs. 5(a), 5(b), and 5(c) can be compared to the experimental asymmetry shown in Fig. 2(a). For the remagnetization process, we find that almost all energies show an almost linear dependence between asymmetry and magnetic moments. Judging from Fig. 5, the remagnetization is consistent with magnon excitations (both short and long wavelengths) and that Stoner excitations are less frequent. Interestingly, the $\mathrm{Ni}$ asymmetries for all three types of excitation shown in Figs. 5(d), 5(e), and 5(f) exhibit no large irregular variations. As for $\mathrm{Fe}$, there are nonlinear contributions at high photon energies, but these asymmetries are too small to be experimentally measured. This correlates well with the measured energy-independent behavior of Ni in Fig. 3(b). Intuitively, an energy-independent behavior would suggest long wavelength magnons to be the dominant excitation process; however, the experimental data is now determined to also support a decreased exchange splitting of $\mathrm{Ni}$, which has been found in other studies [57,58].

As discussed above, a proportionality between asymmetry and magnetization cannot be assumed generally for all possible types of excitations and materials. To highlight the effect of nonlinearities imposed by the relation between $\epsilon_{x y}$ and magnetization, we first show in Fig. 6 the real part of $\epsilon_{x y}$, calculated for various kinds of excitations in the two systems. Overall, this component indeed reflects the magnetization, but for certain excitations the spectral features either shift or even change their shape. These irregularities are even more apparent, if one tracks the evolution of this property at selected energies. We show the real and imaginary parts of $\epsilon_{x y}$ as a function of magnetization at several photon energies in Figs. 7(a)-7(c) and Figs. 7(d) and 7(e) for Fe and $\mathrm{Ni}$, respectively. Here $\epsilon_{x y}$ has been obtained at the energies indicated by the arrows in Figs. 5(a) and 5(d). For Ni, it is clear that an almost proportional relation exists between $\epsilon_{x y}$ and the magnetization for all three types of magnetic excitations. For Fe, there is an approximate proportionality for excitations corresponding to long and short wavelength magnons. For Stoner-type excitations with a collinear decrease of the magnetic moments, there are strong deviations 

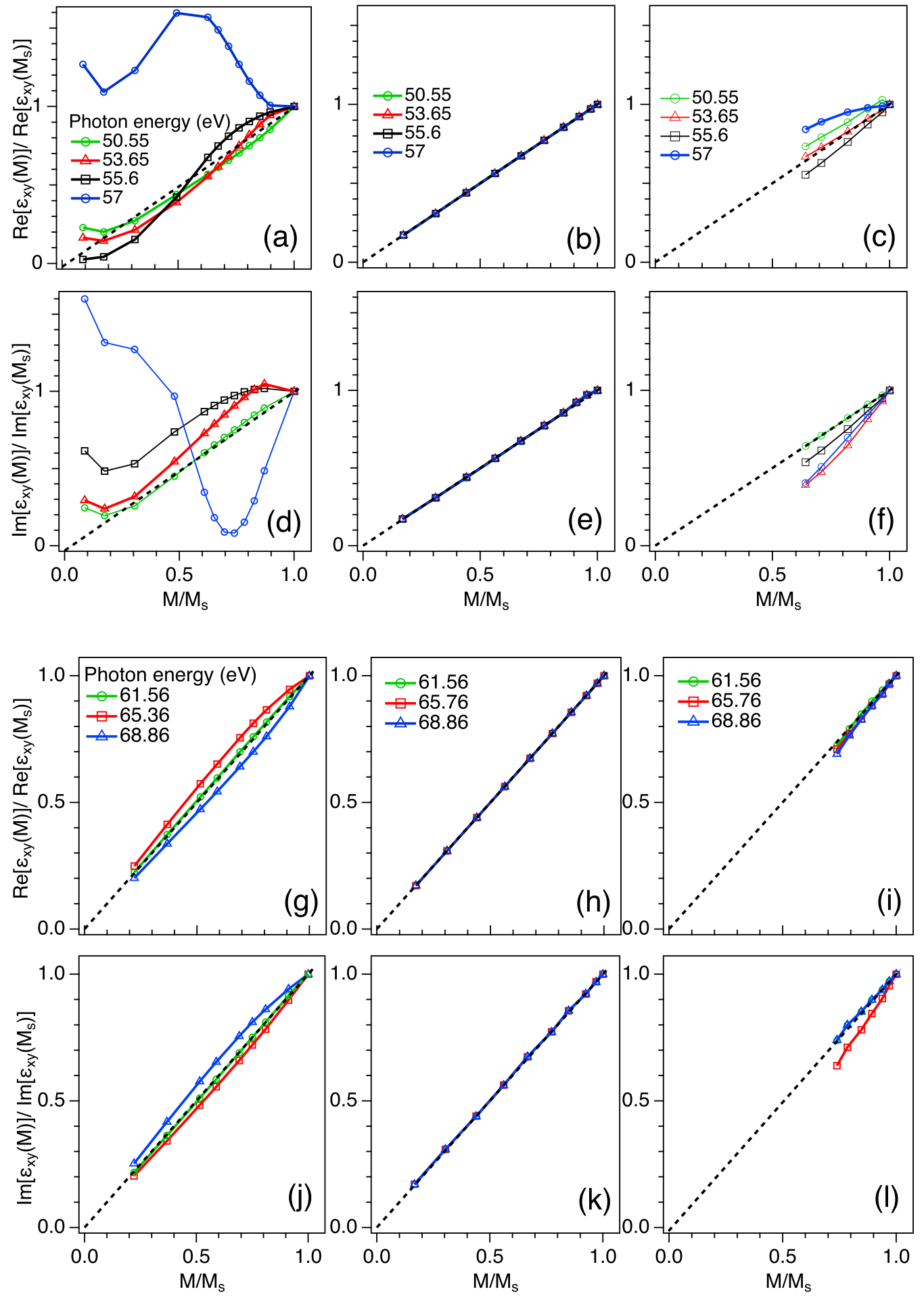

(h)

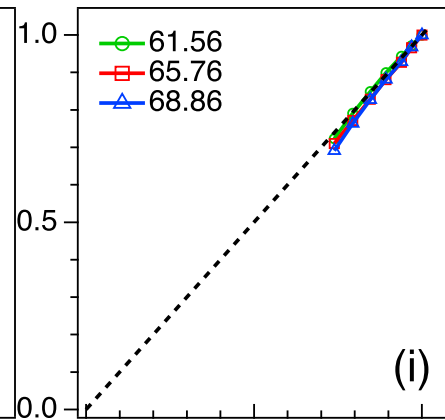

(i)

FIG. 7. Calculated real and imaginary parts of $\epsilon_{x y}$ as a function of reduced magnetization $M / M_{s}$, for Stoner-like excitation in panels (a), (d), (g), and (j), long wavelength magnon excitations in panels (b), (e), (h), and (k) and a high density of short wavelength magnons in panels (c), (f), (i), and (l). The chosen energies are also indicated by arrows in the top panels in Fig. 5. Results for Fe are in panels (a)-(f) and for $\mathrm{Ni}$ in panels (g)-(l). The components of $\epsilon_{x y}$ are normalized with respect to their values obtained for the ferromagnetic ground state (saturated magnetization).

from the proportionality, as shown in Fig. 5. As a matter of fact, such type of nonlinearities between $\mathbf{M}$ and $\epsilon_{x y}$ were predicted theoretically long time ago [23].

Further insight can be obtained by analyzing the relation between the magnetic asymmetry and magnetization in the same way. The corresponding results are shown in Fig. 8. In the case of $\mathrm{Fe}$, one can see that the overall behavior of asymmetry is reminiscent of that of $\epsilon_{x y}$ [Figs. 7(a)-7(f)]. Strongly nonlinear behavior of Fe for the Stoner and short wavelength magnons, already observed for $\epsilon_{x y}$, is further 

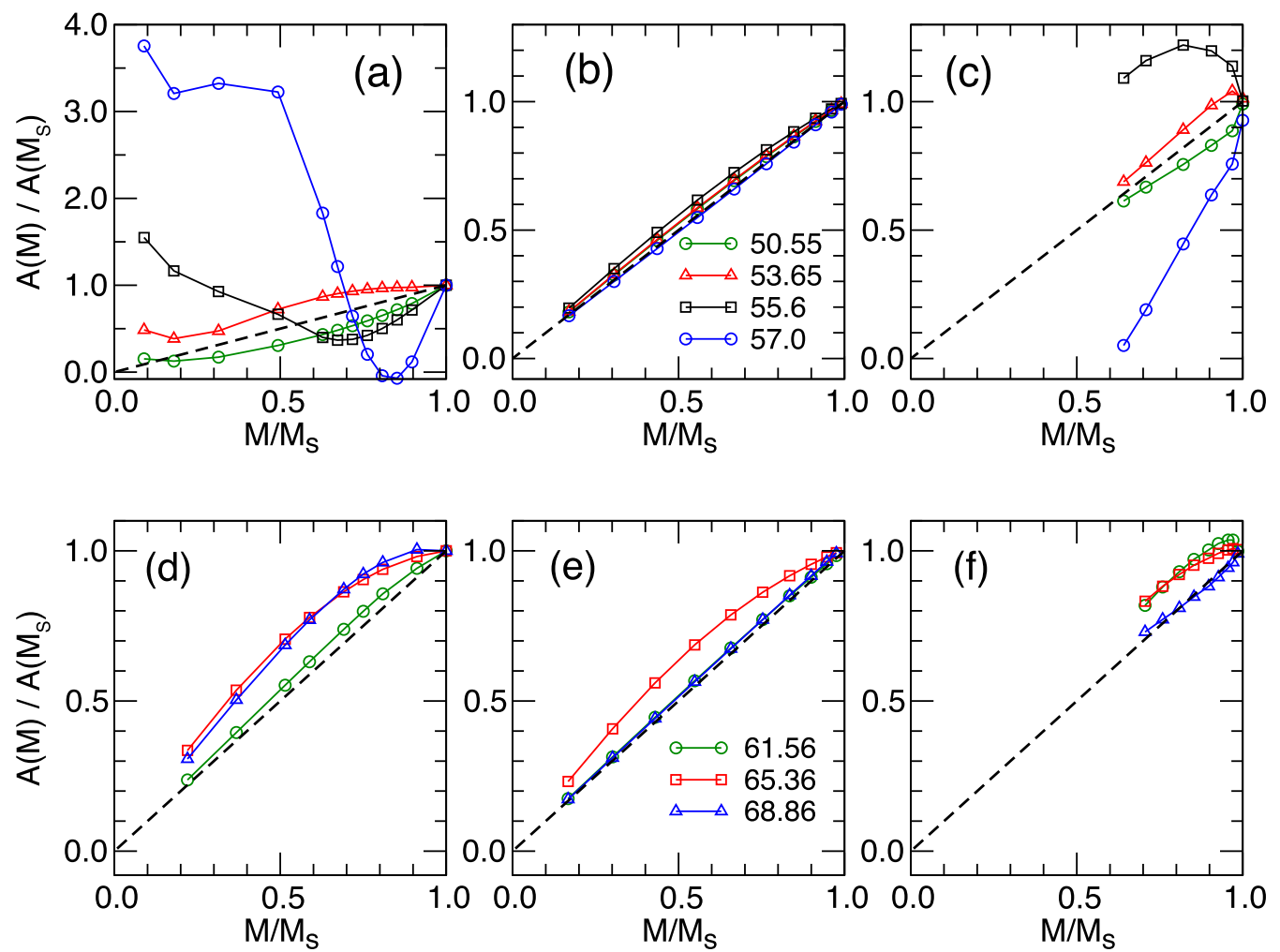

FIG. 8. Calculated magnetic asymmetry as a function of reduced magnetization $M / M_{s}$, for Stoner-like excitation in panels (a) and (d), long wavelength magnon excitations in panels (b) and (e), and a high density of short wavelength magnons in panels (c) and (f). The chosen energies are also indicated by arrows in the top panels in Fig. 5. Results for Fe are in panels (a)-(c) and for Ni in panels (d)-(f). The asymmetries are normalized with respect to their values obtained for the ferromagnetic ground state (saturated magnetization).

enhanced, which is partially related with small changes in the diagonal components of the dielectric tensor (not shown). At the same time, even though the data for Ni show a better proportionality to the magnetization, the nonlinear effects also become apparent. Here, however, these nonlinearities do not originate from the changes in the dielectric tensor, shown above in Figs. 7(g)-7(1). This is best seen for the long wavelength magnons, which are characterized by perfectly linear $\epsilon_{x y}$, yet exhibiting a deviation from proportionality for the magnetic asymmetry at the energies close to the main peak $(65.36 \mathrm{eV})$. The same kind of nonlinearities dominate the spectra for other two types of considered magnetic excitations. Combining the information obtained from Figs. 7 and 8, we are thus able to distinguish the nonlinear effects originating from the intrinsic changes in the band structure and from the breakdown of a linear relation between asymmetry and $\epsilon_{x y}$ [Eq. (2)].

In previous sections, we have shown how various kinds of magnetic excitations will alter the dielectric tensor and the magnetic asymmetry. However, it is not immediately clear which excitations are relevant for different materials and how they would contribute to the optical response. Given that the system is strongly excited, it is not expected to be in a single configuration (microstate). Rather, we expect it to be in a (partially equilibrated) thermal average over a range of excitations. Of all the excited states, only the ones with a sufficient population contribute to the magnetic asymmetry. These are states with an excitation energy above the ground state that are comparable to the average energy per unit cell or the thermal energy (notice that even after a few picoseconds the system of electrons and magnons still retains a rather high effective temperature). Estimated spin temperatures from the three-temperature model [1] gives $\approx 450 \mathrm{~K}$ and $\approx 700 \mathrm{~K}$ for $\mathrm{Ni}$ and $\mathrm{Fe}$, respectively [59], which is about $70 \%$ of the respective magnetic transition temperatures.

Thus, the relative population in principle can be deduced from an analysis of the DFT total energies of the microstates, studied in the previous section. However, a problem appears in practice because the noncollinear configuration which we considered for the case of simulating a high density of short wavelength magnons is limited and only represents a small subset of allowed configurations. Thus, we cannot quantitatively compare the relative energies of these states, and compare them to, e.g., the Stoner excitations.

It is nevertheless worth analyzing results which are obtained for a restricted set of noncollinear states, keeping in mind that they do not provide the complete picture. Our results indicate that in order to reach a small decrease of the magnetization in bcc Fe, Stoner excitations and high-density magnon excitations have similar energies. In order to reach small values of the magnetization $\left(<0.8 M_{s}\right)$, the noncollinear configuration will be energetically preferable compared to reduced values of the moments dictated by Stoner excitations. On the other hand, $\mathrm{Ni}$, being an itinerant ferromagnet with a rather low value of the saturation moment, shows a completely different behavior. Here the Stoner excitations provide the much more efficient channel for the reduction of the magnetization compared to the selected short wavelength 
magnons. These results are in qualitative agreement with recent calculations of the temperature-dependent magnetic properties, where a different behavior of the two systems has been reported [60].

\section{DISCUSSION AND CONCLUSION}

The relationship between sample magnetization and measured asymmetry, obtained from T-MOKE experiments around the $M$-absorption edges of $\mathrm{Fe}$ and $\mathrm{Ni}$, has been investigated using a pump-probe technique. The measured asymmetries have been compared with those obtained from theoretical modeling, showing a good qualitative agreement. Calculations have also been used to determine the relative contributions of three types of magnetic excitations to the variations of asymmetry during the phases of demagnetization and remagnetization.

In Ref. [35], the authors suggest that Stoner and spin wave excitations give distinct fingerprints in the magneto-optical spectra, in agreement with an earlier theoretical proposal [23]. It is worth noting that the ways the Stoner excitations are modeled here (through constrained DFT) and in Ref. [35] (a rigid shift of the majority and minority spin bands) are different. Still, our results indicate that considering highly noncollinear spin states induces yet other types of nonlinearities both in the dielectric tensor and in the magnetic asymmetry and can preclude a clear identification of the main excitation mechanism. In the case of $\mathrm{Ni}$, various types of excitations produce practically indistinguishable modifications in the magnetic asymmetry. At the same time, in the case of Fe, Stoner-type excitations introduce a change of sign of magnetic asymmetry at certain energies, giving a rather clear fingerprint. Since the behaviors of Co considered in Ref. [35] as well as those of Fe and $\mathrm{Ni}$ are all different, we conclude that no general statement on the nature of magnetic excitations in ultrafast experiments can be made.

Experimentally, we found that Fe shows a strong energy dependence of the asymmetry during demagnetization. The origin of this behavior cannot be uniquely determined by comparing the different theoretical models to experimental data. However, it is clear that the out-of-equilibrium situation induced by the pump excitation creates an electronic and magnetic state that has a more complex magneto-optical response than the assumptions that lead to Eq. (3). The time-independent MAT ratios, found for times $t \geqslant 2 \mathrm{ps}$ for $\mathrm{Fe}$, indicate that the remagnetization process must be dominated by magnons in this time range. In contrast, for $\mathrm{Ni}$ the energy dependence of the asymmetry does not show qualitative differences during demagnetization and remagnetization. Theoretical calculations reveal that all considered magnetic excitations result in changes of the off-diagonal component of dielectric tensor, scaling linearly with the magnetization. At the same time, we report that in $\mathrm{Ni}$ there is a nonlinear behavior of asymmetry with respect to $\epsilon_{x y}$, which is especially pronounced at the main peak. This type of nonlinearity is not related to the modifications of the electronic structure.

Overall, the results presented here point out that a direct linear relationship between asymmetry measured in pumpprobe experiments and magnetization cannot be assumed in general. This implies that one needs to interpret these types of experiments with some care and that ideally one should investigate the optical response for more than one photon energy of the probe. Furthermore, our results stress that a deeper insight into the nature of the magnetic excitations of a specific material can be obtained by integrating these types of experiments with a more involved theoretical analysis than usually done.

\section{ACKNOWLEDGMENTS}

The financial support from the Swedish Research Council (VR, Contracts No. 2016-04524 and No. 2013-08316) is gratefully acknowledged. O.E. acknowledges the financial support from the Knut and Alice Wallenberg Foundation (KAW), the Foundation for Strategic Research, Energimyndigheten, STandUPP, and eSSENCE. M.B. acknowledges Nanyang Technological University, NAP-SUG, for funding. I.D.M. acknowledges support by the appointment to the JRG program at the APCTP through the Science and Technology Promotion Fund and Lottery Fund of the Korean Government, as well as support by the Korean local governments, Gyeongsangbuk-do Province and Pohang City. The computational work was performed on resources provided by the Swedish National Infrastructure for Computing (SNIC) at the High Performance Computing Center North (HPC2N), at the National Supercomputer Center (NSC), and at the PDC center for High Performance Computing.
[1] E. Beaurepaire, J.-C. Merle, A. Daunois, and J.-Y. Bigot, Phys. Rev. Lett. 76, 4250 (1996).

[2] B. Koopmans, J. J. M. Ruigrok, F. Dalla Longa, and W. J. M. de Jonge, Phys. Rev. Lett. 95, 267207 (2005).

[3] C. Stamm, T. Kachel, N. Pontius, R. Mitzner, T. Quast, K. Holldack, S. Khan, C. Lupulescu, E. F. Aziz, M. Wietstruk, H. A. Dürr, and W. Eberhardt, Nat. Mater. 6, 740 (2007).

[4] M. Krauss, T. Roth, S. Alebrand, D. Steil, M. Cinchetti, M. Aeschlimann, and H. C. Schneider, Phys. Rev. B 80, 180407(R) (2009).

[5] J.-Y. Bigot, M. Vomir, and E. Beaurepaire, Nat. Phys. 5, 515 (2009).
[6] B. Koopmans, G. Malinowski, F. Dalla Longa, D. Steiauf, M. Faehnle, T. Roth, M. Cinchetti, and M. Aeschlimann, Nat. Mater. 9, 259 (2010).

[7] K. Carva, M. Battiato, and P. M. Oppeneer, Phys. Rev. Lett. 107, 207201 (2011).

[8] D. Rudolf, C. La-O-Vorakiat, M. Battiato, R. Adam, J. M. Shaw, E. Turgut, P. Maldonado, S. Mathias, P. Grychtol, H. T. Nembach, T. J. Silva, M. Aeschlimann, H. C. Kapteyn, M. M. Murnane, C. M. Schneider, and P. M. Oppeneer, Nat. Commun. 3, 1037 (2012).

[9] C. E. Graves, A. H. Reid, T. Wang, B. Wu, S. de Jong, K. Vahaplar, I. Radu, D. P. Bernstein, M. Messerschmidt, 
L. Müller, R. Coffee, M. Bionta, S. W. Epp, R. Hartmann, N. Kimmel, G. Hauser, A. Hartmann, P. Holl, H. Gorke, J. H. Mentink, A. Tsukamoto, A. Fognini, J. J. Turner, W. F. Schlotter, D. Rolles, H. Soltau, L. Strüder, Y. Acremann, A. V. Kimel, A. Kirilyuk, T. Rasing, J. Stöhr, A. O. Scherz, and H. A. Dürr, Nat. Mater. 12, 293 (2013).

[10] K. Carva, M. Battiato, D. Legut, and P. M. Oppeneer, Phys. Rev. B 87, 184425 (2013).

[11] M. Battiato, P. Maldonado, and P. M. Oppeneer, J. Appl. Phys. 115, 172611 (2014).

[12] A. J. Schellekens, K. C. Kuiper, R. R. J. C. de Wit, and B. Koopmans, Nat. Commun. 5, 4333 (2014).

[13] W. Tows and G. M. Pastor, Phys. Rev. Lett. 115, 217204 (2015).

[14] M. Elyasi and H. Yang, Phys. Rev. B 94, 024417 (2016).

[15] D. M. Nenno, S. Kaltenborn, and H. C. Schneider, Phys. Rev. B 94, 115102 (2016).

[16] C. D. Stanciu, F. Hansteen, A. V. Kimel, A. Kirilyuk, A. Tsukamoto, A. Itoh, and T. Rasing, Phys. Rev. Lett. 99, 047601 (2007).

[17] A. Kirilyuk, A. V. Kimel, and T. Rasing, Rev. Mod. Phys. 82, 2731 (2010).

[18] G. Malinowski, F. Dalla Longa, J. H. H. Rietjens, P. V. Paluskar, R. Huijink, H. J. M. Swagten, and B. Koopmans, Nat. Phys. 4, 855 (2008)

[19] M. Battiato, K. Carva, and P. M. Oppeneer, Phys. Rev. Lett. 105, 027203 (2010).

[20] A. Melnikov, I. Razdolski, T. O. Wehling, E. T. Papaioannou, V. Roddatis, P. Fumagalli, O. Aktsipetrov, A. I. Lichtenstein, and U. Bovensiepen, Phys. Rev. Lett. 107, 076601 (2011).

[21] A. Eschenlohr, M. Battiato, P. Maldonado, N. Pontius, T. Kachel, K. Holldack, R. Mitzner, A. Föhlisch, P. M. Oppeneer, and C. Stamm, Nat. Mater. 12, 332 (2013).

[22] M. Battiato and K. Held, Phys. Rev. Lett. 116, 196601 (2016).

[23] J. L. Erskine and E. A. Stern, Phys. Rev. B 12, 5016 (1975).

[24] B. Koopmans, M. van Kampen, J. T. Kohlhepp, and W. J. M. de Jonge, Phys. Rev. Lett. 85, 844 (2000).

[25] L. Guidoni, E. Beaurepaire, and J.-Y. Bigot, Phys. Rev. Lett. 89, 017401 (2002).

[26] J.-Y. Bigot, L. Guidoni, E. Beaurepaire, and P. N. Saeta, Phys. Rev. Lett. 93, 077401 (2004).

[27] E. Carpene, E. Mancini, C. Dallera, M. Brenna, E. Puppin, and S. De Silvestri, Phys. Rev. B 78, 174422 (2008).

[28] G. P. Zhang, W. Hübner, G. Lefkidis, Y. Bai, and T. F. George, Nat. Phys. 5, 499 (2009).

[29] E. Carpene, H. Hedayat, F. Boschini, and C. Dallera, Phys. Rev. B 91, 174414 (2015).

[30] C. La-O-Vorakiat, E. Turgut, C. A. Teale, H. C. Kapteyn, M. M. Murnane, S. Mathias, M. Aeschlimann, C. M. Schneider, J. M. Shaw, H. T. Nembach, and T. J. Silva, Phys. Rev. X 2, 011005 (2012).

[31] I. L. M. Locht, I. Di Marco, S. Garnerone, A. Delin, and M. Battiato, Phys. Rev. B 92, 064403 (2015).

[32] P. Oppeneer, Handbook of Magnetic Materials, edited by K. H. J. Buschow (Elsevier, Amsterdam, 2001), Vol. 13.

[33] S. Mathias, C. La-O-Vorakiat, P. Grychtol, P. Granitzka, E. Turgut, J. M. Shaw, R. Adam, H. T. Nembach, M. E. Siemens, S. Eich, C. M. Schneider, T. J. Silva, M. Aeschlimann, M. M. Murnane, and H. C. Kapteyn, Proc. Natl. Acad. Sci. USA 109, 4792 (2012).
[34] S. Mathias, C. La-O-Vorakiat, J. M. Shaw, E. Turgut, P. Grychtol, R. Adam, D. Rudolf, H. T. Nembach, T. J. Silva, M. Aeschlimann, C. M. Schneider, H. C. Kapteyn, and M. M. Murnane, J. Electron. Spectrosc. Relat. Phenom. 189, 164 (2013).

[35] E. Turgut, D. Zusin, D. Legut, K. Carva, R. Knut, J. M. Shaw, C. Chen, Z. Tao, H. T. Nembach, T. J. Silva et al., Phys. Rev. B 94, 220408(R) (2016).

[36] D. Zusin, P. M. Tengdin, M. Gopalakrishnan, C. Gentry, A. Blonsky, M. Gerrity, D. Legut, J. M. Shaw, H. T. Nembach, T. J. Silva, P. M. Oppeneer, H. C. Kapteyn, and M. M. Murnane, Phys. Rev. B 97, 024433 (2018).

[37] S. Eich, M. Plötzing, M. Rollinger, S. Emmerich, R. Adam, C. Chen, H. C. Kapteyn, M. M. Murnane, L. Plucinski, D. Steil, B. Stadtmüller, M. Cinchetti, M. Aeschlimann, C. M. Schneider, and S. Mathias, Sci. Adv. 3, e1602094 (2017).

[38] R. Chimata, E. K. Delczeg-Czirjak, A. Szilva, R. Cardias, Y. O. Kvashnin, M. Pereiro, S. Mankovsky, H. Ebert, D. Thonig, B. Sanyal, A. B. Klautau, and O. Eriksson, Phys. Rev. B 95 , 214417 (2017).

[39] O. Eriksson, A. Bergman, L. Bergqvist, and J. Hellsvik, Atomistic Spin Dynamics: Foundations and Applications, 1st ed. (Oxford University Press, Oxford, UK, 2017).

[40] R. Chimata, A. Bergman, L. Bergqvist, B. Sanyal, and O. Eriksson, Phys. Rev. Lett. 109, 157201 (2012).

[41] E. Runge and E. K. U. Gross, Phys. Rev. Lett. 52, 997 (1984).

[42] K. Krieger, J. K. Dewhurst, P. Elliott, S. Sharma, and E. K. U. Gross, J. Chem. Theory Comput. 11, 4870 (2015).

[43] V. Shokeen, M. Sanchez Piaia, J.-Y. Bigot, T. Müller, P. Elliott, J. K. Dewhurst, S. Sharma, and E. K. U. Gross, Phys. Rev. Lett. 119, 107203 (2017).

[44] K. Krieger, P. Elliott, T. Müller, N. Singh, J. K. Dewhurst, E. K. U. Gross, and S. Sharma, J. Phys.: Condens. Matter 29, 224001 (2017).

[45] S. Plogmaker, J. A. Terschlüsen, N. Krebs, M. Svanqvist, J. Forsberg, U. B. Cappel, J.-E. Rubensson, H. Siegbahn, and J. Söderström, Rev. Sci. Instrum. 86, 123107 (2015).

[46] R. Stefanuik, R. Knut, S. Jana, J. Terschlüsen, A. Sandell, and J. Söderström, J. Electron. Spectrosc. Relat. Phenom. 224, 33 (2018).

[47] S. Jana, J. A. Terschlüsen, R. Stefanuik, S. Plogmaker, S. Troisi, R. S. Malik, M. Svanqvist, R. Knut, J. Söderström, and O. Karis, Rev. Sci. Instrum. 88, 033113 (2017).

[48] X. He, J. M. Dahlström, R. Rakowski, C. M. Heyl, A. Persson, J. Mauritsson, and A. L'Huillier, Phys. Rev. A 82, 033410 (2010).

[49] P. Holloway and J. Hudson, Surf. Sci. 43, 123 (1974).

[50] G. T. Tyuliev and K. L. Kostov, Phys. Rev. B 60, 2900 (1999).

[51] S. Valencia, A. Kleibert, A. Gaupp, J. Rusz, D. Legut, J. Bansmann, W. Gudat, and P. M. Oppeneer, Phys. Rev. Lett. 104, 187401 (2010).

[52] P. Yeh, Surf. Sci. 96, 41 (1980).

[53] http://elk.sourceforge.net/.

[54] J. P. Perdew, K. Burke, and M. Ernzerhof, Phys. Rev. Lett. 77, 3865 (1996).

[55] U. von Barth and L. Hedin, J. Phys. C: Solid State Phys. 5, 1629 (1972).

[56] F. Willems, S. Sharma, C. v. Korff Schmising, J. K. Dewhurst, L. Salemi, D. Schick, P. Hessing, C. Strüber, W. D. Engel, and S. Eisebitt, Phys. Rev. Lett. 122, 217202 (2019). 
[57] H.-S. Rhie, H. A. Dürr, and W. Eberhardt, Phys. Rev. Lett. 90, 247201 (2003).

[58] B. Y. Mueller, A. Baral, S. Vollmar, M. Cinchetti, M. Aeschlimann, H. C. Schneider, and B. Rethfeld, Phys. Rev. Lett. 111, 167204 (2013).
[59] K. Thurnay, Forschungszentrum Karlsruhe, Technik und Umwelt, Wissenschaftliche Berichte FZKA 6095, Tech. Rep. (Forschungszentrum Karlsruhe GmbH, Karsruhe, 1998).

[60] F. Pan, J. Chico, A. Delin, A. Bergman, and L. Bergqvist, Phys. Rev. B 95, 184432 (2017). 